\title{
The 3D structure of a complex quasicrystal approximant determined by electron crystallography
}

\author{
Xiaodong Zou*, Zhimin Mo*, Sven Hovmöller*, Xingzhong Li** and Kehsin $\mathrm{Kuo}^{* * *}$ \\ *Structural Chemistry, Stockholm University, SE-106 91 Stockholm, Sweden \\ **Center for Materials Research \& Analysis, Univ. of Nebraska-Lincoln, Lincoln, NE 68588-0656 \\ ***Beijing Laboratory of Electron Microscopy, P.O. Box 2724, 100080 Beijing, China
}

Electron crystallography can be applied for solving structures of crystals too small f or X -ray diffraction. The advantage of electron crystallography over X -ray crystallography is that the phases of the crystallographic structure factors, which are lost in X -ray diffraction, are present in HREM images. Electron crystallography has been demo nstrated on many structures with one short axis $[1,2]$. However, for crystals lacking of short axes, atoms may overlap in any directions so several images from different directions must be combined in order to solve the 3D structure. One of such examples was shown on a mineral staurolite [3]. Here the 3D reconstruction is applied to a very complex quasicrystal approximant - $\mathrm{V}$-AlCrFe with the space group P6 ${ }_{3} / \mathrm{m}$ and $a=40.687$ and $c=$ $12.546 \AA$. The structure was too complicated to be solved directly by s ingle crystal X-ray diffraction and part of the structure was first deduced from the related phases by comparing the HREM images. A complete structure model was then obtained from difference Fourier maps and refined by single crystal X-ray diffraction [4].

HREM images and electron diffraction patterns of the $v$-AlCrFe phase were collected from different zone axes and digitised by a CCD camera (Fig. 1). For each image, Fourier transform was calculated from the thinnest part of the crystal using the program C RISP (Fig. 2). The defocus value and astigmatism under which the image was taken were estimated from the position(s) of the dark $\operatorname{ring}(\mathrm{s})$ in the Fourier transform and their effects were compensated for. Amplitude and phase were extracted from each reflectio $n$ and the symmetry restrictions were then applied. Finally a potential map was calculated by inverse Fourier transformation using the corrected amplitudes and phases (Fig. 2c). Intensities of electron diffraction spots were extracted using the program ELD.

Amplitudes and phases from 11 zone axes were merged into a 3D data set. Amplitudes from the HREM images were replaced by those from the ED patterns. A 3D potential map was calculated from the 3D data by XtalView. Atoms are clearly resolved from the map (Fig. 3). The structure is layered and contains one unique flat layer $(\mathrm{F})$ and one unique puckered $(\mathrm{P})$ layer. In each unit cell, six such layers are stacked along the $c$-axis, with the stacking sequence $\mathrm{PFP}^{\mathrm{m}}\left(\mathrm{PFP}^{\mathrm{m}}\right)^{\prime}$, where $\mathrm{P}$ and $\mathrm{P}^{\mathrm{m}}$ are related by a mirror, $\mathrm{PFP}^{\mathrm{m}}$ and $\left(\mathrm{PFP}^{\mathrm{m}}\right)$ ' are related by a $2_{1}$ axis. 136 unique atomic positions could be located from the two unique layers. The structure obtained from 3D reconstruction is very similar to that obtained by X-ray diffraction [4].

References

1. Weirich, T.E., Ramlau, R., Simon, A., Hovmöller, S. \& Zou, X.D. (1996) Nature, 382, 144-146.

2. Zou, X.D., Sundberg, M., Larine, M. \& Hovmöller, S. (1996) Ultramicroscopy, 62, 103-121.

3. Wenk, H.-R., Downing, K.H., Meisheng, H. \& O’Keefe, M.A. (1992) Acta Cryst. A48, 700-716.

4. Mo, Z.M., Zhou, H.Y. \& Kuo, K.H. (2000) Acta Cryst. B56, 392-401. 


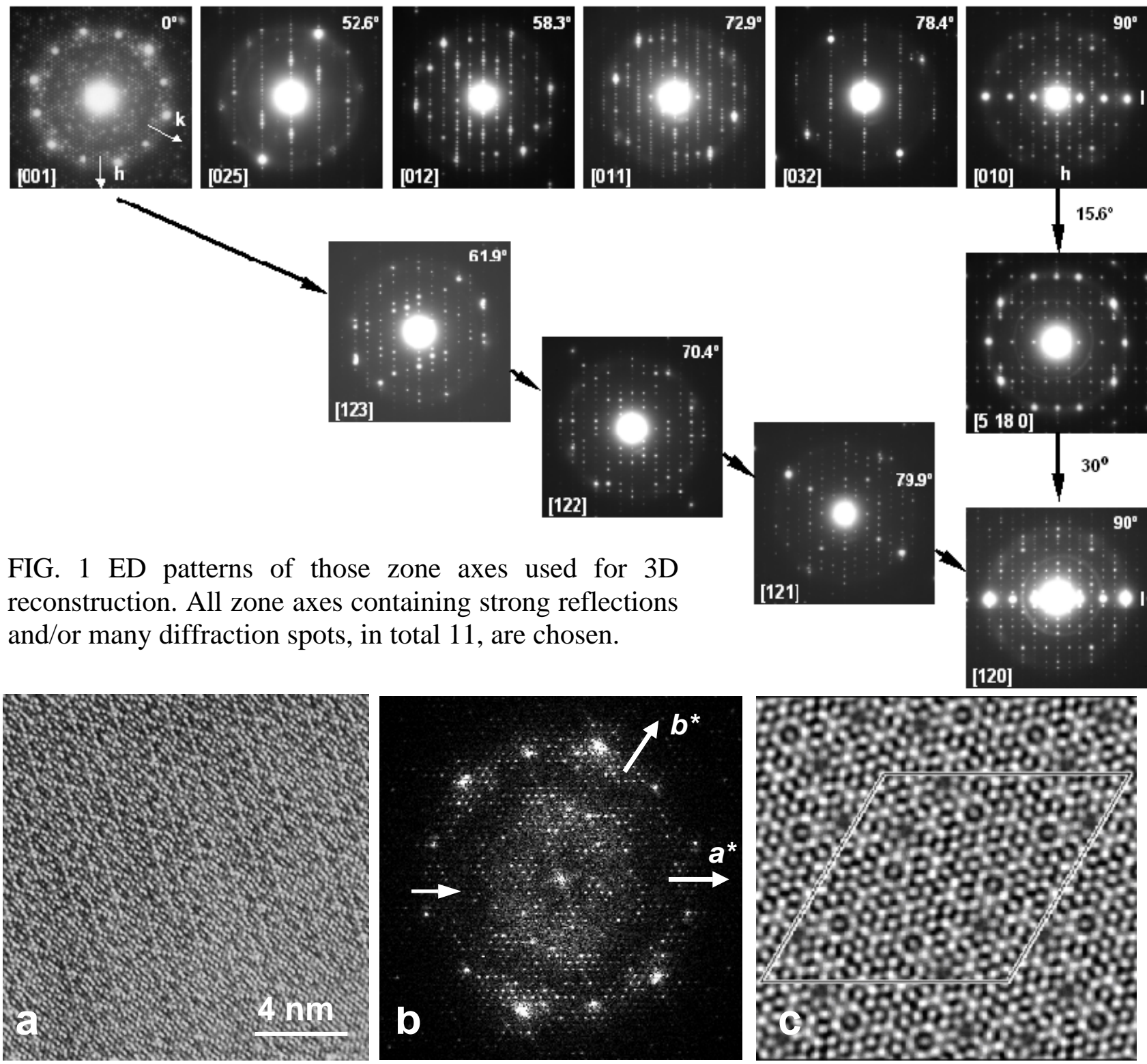

FIG. 2 a) A [001] HREM image of the v-AlFeCr phase and b) its Fourier transform. The defocus was determined from the dark ring (marked by an arrow) that corresponds $t$ o the zero cross -over. c) Projected potential maps after imposing the symmetry and compensating for the CTF.
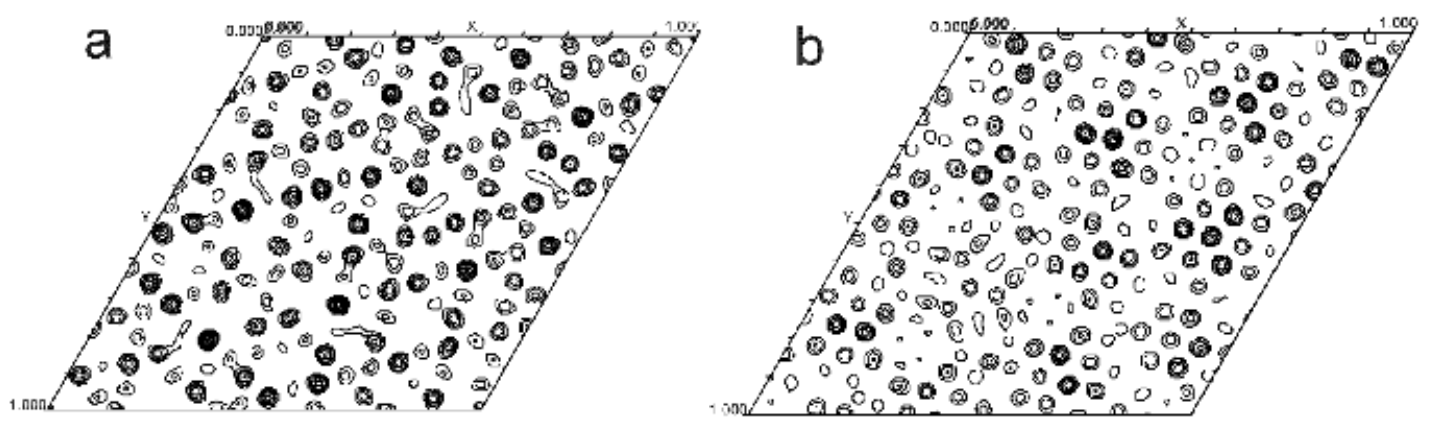

FIG. 3 Sections of the 3D potential map obtained from the 3D reconstruction. a) The flat layer $(F)$ at $\mathrm{z}=0.25$ and $\mathrm{b}$ ) the puckered layer $(\mathrm{P})$ with $\mathrm{z}=0.35-0.45$. 